\title{
Consecutive-Day Intake of Whey Protein Upregulates mTOR mRNA and Protein Expression in Resting Skeletal Muscle of Mice
}

\author{
Hajime Sasaki \\ Department of Nutrition and Life Sciences, Kanagawa-Institute of Technology, Atsugi, Japan \\ Email: sasaki@cco.kanagawa-it.ac.jp
}

How to cite this paper: Sasaki, H. (2019) Consecutive-Day Intake of Whey Protein Upregulates mTOR mRNA and Protein Expression in Resting Skeletal Muscle of Mice. Food and Nutrition Sciences, 10 , 1035-1044.

https://doi.org/10.4236/fns.2019.109074

Received: August 6, 2019

Accepted: August 31, 2019

Published: September 3, 2019

Copyright ( 2019 by author(s) and Scientific Research Publishing Inc. This work is licensed under the Creative Commons Attribution International License (CC BY 4.0).

http://creativecommons.org/licenses/by/4.0/

\begin{abstract}
Background: Although the effect of whey protein intake on protein metabolism in exercise-loaded skeletal muscle has been well documented, little has been reported on its effect on resting muscle. The effects of whey protein intake on protein metabolism in resting mouse skeletal muscle were investigated. Methods: Mice were fed AIN-93G composed of either casein or whey protein as the protein source for 3 or 7 consecutive days. The gastrocnemius muscle was excised, and the expression levels of the regulatory factor, mTOR, and its subunits, Raptor and Rictor, were measured by real-time PCR. The protein expression levels of mTOR and its phosphorylated form were measured by immunofluorescent western blotting. The effects of whey protein were compared to those of the case in control. Results: mTOR expression increased in the gastrocnemius muscle of mice fed whey protein for 7 consecutive days. The expression of Raptor significantly increased, whereas that of Rictor did not change, suggesting a dominant formation of mTORC1 relating to the upregulation of protein synthesis. The protein levels of mTOR and its phosphorylated form significantly increased in mice fed whey protein, indicating enhanced protein synthesis. Increased mTOR expression was not seen in the gastrocnemius muscle of mice fed whey protein for 3 consecutive days. Conclusions: These results indicate that the intake of whey protein for 7 consecutive days, but not 3 days, upregulates the mRNA and protein expression of mTOR in the resting gastrocnemius muscle of mice, suggesting its ability to enhance protein synthesis. Consecutive-day intake of whey protein may induce constitutive alteration of the skeletal muscle, including continuous upregulation of muscle protein synthesis.
\end{abstract}

\section{Keywords}

Whey Protein Intake, Resting Skeletal Muscle, Enhancement, Protein 
Synthesis, mTOR Pathway

\section{Introduction}

Sarcopenia is an age-related loss of muscle mass and quality [1], which is thought to be caused by combined age-related incidences including decreased protein supply due to reduced food intake, enhanced protein breakdown induced by insulin resistance, decreased potency of protein synthesis, and persistent chronic low-grade inflammation caused by aging [2]. A loss of muscle impacts not only physical body movements but also whole-body metabolic homeostasis, as muscles are an endocrine organ in which hormones and cytokines produced by myocytes affect the whole-body physiology [3]. Additionally, decline of these muscle functions results in age-related adverse outcomes, frailty, and shortened life expectancy [4] [5].

Regular exercise and dietary intervention are more effective than pharmacological therapy in the prevention of sarcopenia [6]. Exercise is thought to induce physiological changes in muscles, such as repeated transient mRNA production [7], followed by post-exercise responses that activate myogenic and metabolic events, wherein protein supply is necessary. Post-exercise dietary intervention to supply protein is required to fulfill this prerequisite to enhance protein production.

Exercise-induced increase in muscle protein synthesis is regulated by mechanistic target of rapamycin complex 1 (mTORC1), a serine/threonine protein kinase, which plays a pivotal role in the regulation of protein synthesis [8]. In fact, exercise induces mTORC1, thereby stimulating protein synthesis in skeletal muscles [9], and dietary protein intake supplies the amino acids required for this mTOR-activated protein synthesis leading to protein anabolism.

Because whey protein is a protein source with nutritionally complete amino acid composition, it is often used as a post-exercise protein supplement. Whey protein is suggested to more potently enhance post-exercise protein synthesis than casein or soy protein [10] [11], indicating that there may be a mechanism to recognize whey protein in skeletal muscle. Moreover, whey protein transiently enhances protein synthesis in resting skeletal muscle, even though its enhanced level is significantly lower than that in the exercise-loaded muscle [11]. These facts suggest that both an exercise activatable pathway and a whey protein activatable pathway are involved in the post-exercise effect of whey protein in enhancing protein synthesis.

This study describes that, compared to casein, the consecutive intake of whey protein over 7 days potently increases the mRNA expression of mTORC1, together with an increase in its protein expression in the resting skeletal muscles of mice, which is distinct from the transient effect of a 3-day consecutive intake of whey protein. The results suggest that there may be cellular machinery that continuously enhances protein synthesis in response to an extended intake of whey 
protein, while transiently responding to a bout of whey protein intake. These results offer a basis for whey protein to be the better candidate in dietary therapy for clinical problems, including sarcopenia, where an anabolic balance in muscle protein metabolism is preferred.

\section{Materials and Methods}

\subsection{Animal Experiments}

Animal experiments were conducted in accordance with the Guidelines for Proper Conduct of Animals Experiments (The Science Council of Japan) after approval from the Animal Ethical Care Committee of Kanagawa Institute of Technology.

Six-week-old male C57BL mice were purchased from Charles River Laboratories (Japan) and separated into two groups $(n=5)$. They were fed AIN-93G, comprising of casein (Oriental Yeast, Japan) as the protein source for 5 days to acclimate them to the environment and diet. Then, they were fed AIN-93G composed of either casein or whey protein (whey protein isolate, Arla Foods Ingredients, Japan) as the protein source for 1 week. The mice were maintained at constant room temperature, $20^{\circ} \mathrm{C}-22^{\circ} \mathrm{C}$, with free access to water and diet under a 12:12-h light-dark cycle with lights on at 7:00 AM.

The gastrocnemius muscle was excised and then immersed and stored in RNA Later (Thermo Fischer Scientific, USA) at $-20^{\circ} \mathrm{C}$.

\subsection{Real-Time PCR}

Total RNA was extracted from tissues using the ReliaPrep RNA Miniprep Systems (Promega, USA) following the manufacturer's instructions. Reverse transcription of RNA to cDNA was performed using ReverTra Ace qPCR RT Master Mix (TOYOBO, Japan) on the ABI Geneamp 9700 PCR-Thermal Cycler (Applied Biosystems, USA). Real-time PCR was performed using KOD-Plus-Ver. 2 (TOYOBO, Japan) on the ABI Applied Biosystems 7300 Real-Time PCR System (Applied Biosystems, USA). Primers used to detect and quantify gene expression were synthesized by TAKARA (Japan). Their sequences are shown in Table 1.

Table 1. List of DNA primer sets for quantitative real-time PCR.

\begin{tabular}{|c|c|c|c|c|c|}
\hline Gene & Direction & Sequence & $\begin{array}{c}\text { Start } \\
\text { position }\end{array}$ & Size & $\begin{array}{l}\text { Variant } \\
\text { coverage }\end{array}$ \\
\hline \multirow{2}{*}{$18 \mathrm{~S}$ rRNA } & Forward & TTCTGGCCAACGGTCTAGACAAC & 314 & \multirow{2}{*}{127} & \multirow{2}{*}{ - } \\
\hline & Reverse & CCAGTGGTCTTGGTGTGCTGA & 440 & & \\
\hline \multirow{2}{*}{ mTOR } & Forward & CCCGGACAAGGACAGACTCCTA & 7445 & \multirow{2}{*}{145} & \multirow{2}{*}{ - } \\
\hline & Reverse & GGTTTCACCAAACCGTCTCCA & 7589 & & \\
\hline \multirow{2}{*}{ Raptor } & Forward & CATCAGCTCTGTTGATGGTGGAA & 5905 & \multirow{2}{*}{80} & \multirow{2}{*}{$2 / 2$} \\
\hline & Reverse & GCACACCAGGACACTGTGGAA & 5984 & & \\
\hline \multirow{2}{*}{ Rictor } & Forward & TCTTGTCCAGAGCTTGAGACAGTTG & 7988 & \multirow{2}{*}{115} & \multirow{2}{*}{ - } \\
\hline & Reverse & GGCTGAGAGTGCATGGAAACA & 8102 & & \\
\hline
\end{tabular}


The levels of the genes of interest were normalized to the level of the internal control gene, $18 \mathrm{~S}$ rRNA, and are expressed as the mean with the standard error of the mean in the figures.

\subsection{Western Blotting}

First, $10 \mathrm{mg}$ gastrocnemius muscle was homogenized in $90 \mu \mathrm{L}$ lysis buffer in a plastic test tube on ice using a micro pestle (BioMasher, TAKARA, Japan). The supernatant obtained after centrifugation at $14,000 \mathrm{rpm}$ for $10 \mathrm{~min}$ at $4^{\circ} \mathrm{C}$ was used as the protein extract. The lysis buffer was constituted with $20 \mathrm{mM}$ Tris$\mathrm{HCl}$ (pH 7.4), $150 \mathrm{mM} \mathrm{NaCl}, 1 \%$ Triton X-100, phosphatase inhibitors (10 mM Na-pyrophosphate, $25 \mathrm{mM} \mathrm{NaF}, 5 \mathrm{mM} \mathrm{Na}_{3} \mathrm{VO}_{4}$, and $10 \mathrm{mM}$ EDTA-2Na) and $1 \%$ ProteoGuard EDTA-Free Protease Inhibitor Cocktail (ClonTech, USA). The amount of protein in the supernatant was measured by the BCA method. To resolve the protein, $10 \mu \mathrm{g}$ extracted protein in SDS electrophoresis sample buffer was loaded onto an SDS polyacrylamide gradient gel (10\% - 20\%). After electrophoresis, the proteins were transferred to a PVDF membrane using the semidry blotting method. mTOR or phosphorylated mTOR were detected using primary antibodies, an HRP-conjugated secondary antibody and a chemiluminescent probe. The primary antibodies used were anti-mTOR (2972S, Cell Signaling, USA) and anti-phospho-mTOR (Ser 2448) (2974S, Cell Signaling, USA). The signal intensity was analyzed using Image J (NHI, USA).

\subsection{Statistical Analysis}

The results were statistically analyzed using a Student's t-test.

\section{Results}

Body weights of mice fed casein or whey protein at the end of 7 days were $21.0 \pm$ $0.2 \mathrm{~g}$ and $21.3 \pm 0.4 \mathrm{~g}$ (mean \pm standard error), respectively, and no significant difference was noted.

mTOR comprises two distinct complexes, mTORC1 and mTORC2. The key subunits distinct between mTORC1 and mTORC2 are Raptor (regulatory protein associated with mTOR) in mTORC1 and Rictor (rapamycin insensitive companion of mTOR) in mTORC2 [12] [13] [14] [15]. To determine which mTOR complex was dominant, the mRNA levels of both Raptor and Rictor were measured together with that of mTOR in mice fed with casein or whey protein for 7 days.

The mRNA level of mTOR in the gastrocnemius muscle was significantly higher in mice fed whey protein than in mice fed casein (Figure 1). The expression of Raptor was significantly higher in whey fed mice, whereas no significant difference was seen in the mRNA levels of Rictor between mice fed whey protein or casein (Figure 2).

The protein level of mTOR was detected by a chemiluminescent method following immunoblotting. Chemiluminescent signals of mTOR were higher in mice fed whey protein than in mice fed casein (Figure 3). 


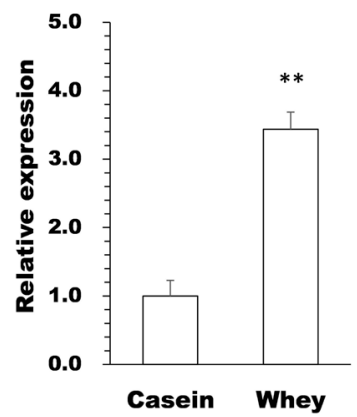

Figure 1. Effect of whey protein on mTOR mRNA level in mouse gastrocnemius muscle. The mRNA level was normalized to that in the case in control. Whey: whey protein. ${ }^{* *}: \mathrm{P}<0.01$.
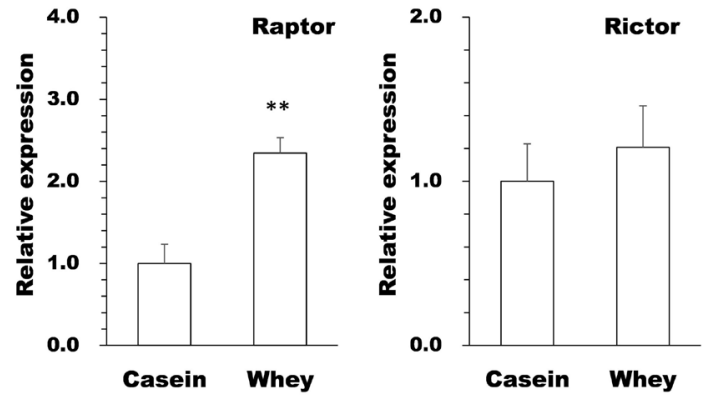

Figure 2. Effect of whey protein on mRNA levels of Raptor and Rictor in mouse gastrocnemius muscle. The mRNA levels were normalized to those in the case in control. Whey: whey protein. ${ }^{* *}$ : $\mathrm{P}<0.01$.
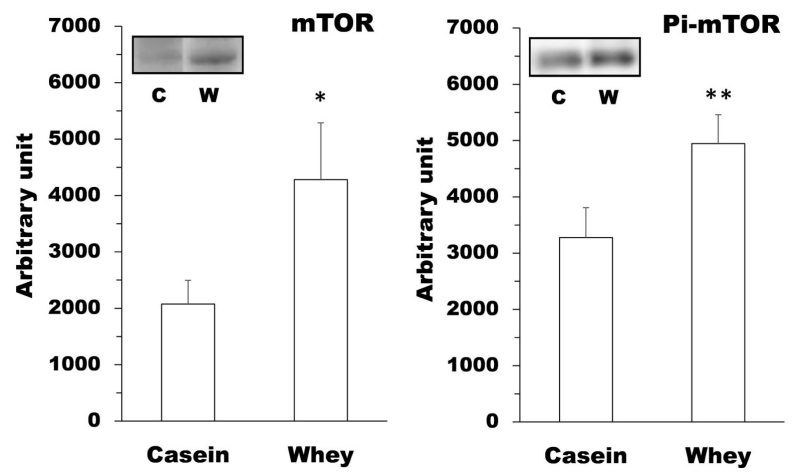

Figure 3. Effect of whey protein on the protein levels of mTOR and phosphorylated mTOR (Pi-mTOR) in mouse gastrocnemius muscle. Inserts are the images of proteins on the blotted membrane detected by immunofluorescence. Whey: whey protein. C: casein, $\mathrm{W}$ : whey protein. ${ }^{*}: \mathrm{P}<0.05,{ }^{* *}$ : $\mathrm{P}<0.01$.

Since mTOR is activated by phosphorylation, levels of phosphorylated mTOR were measured using the same method. The signal intensity was significantly higher in mice fed whey protein than in mice fed casein (Figure 3).

The mRNA levels were measured in the gastrocnemius muscle excised from mice after 3 or 7 consecutive days of feeding whey or casein. The mRNA level of mTOR in mice fed whey protein did not increase after the 3-day feeding, but significantly increased after the 7-day feeding (Figure 4). 


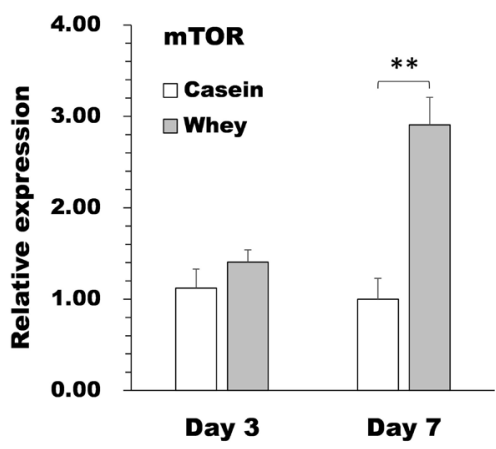

Figure 4. mTOR mRNA levels in the gastrocnemius muscle of mice fed whey protein for 3 days (Day 3 ) or 7 days (Day 7). The mRNA levels were normalized to that in the casein control at Day $7 .{ }^{* *}$ : $\mathrm{P}<0.01$.

\section{Discussion}

This study determined that, compared to casein, the consecutive-day intake of whey protein affects the resting gastrocnemius muscle, and transcriptionally enhances the gene expression related to the cellular machinery for protein synthesis. Namely, it enhanced the mRNA expression of mTOR and Raptor in the resting gastrocnemius muscle of mice, indicating the dominant formation of mTORC1. Moreover, it increased levels of both mTOR protein and its phosphorylated form. These results suggest that the consecutive-day intake of whey protein chronically enhanced protein synthesis by activating mTORC1 pathways in resting muscles

Although little has been reported on the effects of protein intake on resting muscles, its effects on muscles after exercise have been well documented. Exercise activates both protein breakdown and protein synthesis in skeletal muscle. If protein is not supplied after exercise, muscle breakdown surpasses protein synthesis [16] [17] [18]. Post-exercise protein intake affects this muscle catabolism/anabolism balance, resulting in anabolic metabolism [19]. In accordance with this diet-induced change in the metabolic balance, the expression of mTOR, a primary regulator of protein synthesis, is downregulated by exercise, and upregulated by post-exercise intake of whey protein [16] [20].

A short period of whey protein intake after resistance exercise transiently enhances protein synthesis. The muscle response to protein intake becomes most potent 1 to 2 hours post-exercise and continues up to 4 hours [21]. This timecourse profile is attributed partly to the response of muscles to the growth hormones that are secreted by exercise-loaded muscles. In fact, growth hormone secretion precedes the time period for muscles to respond to protein intake and the time-course profile of its secretion parallels the muscle response. In fact, exercise-loaded muscles transiently secrete growth hormones into the bloodstream, reaching a maximal blood concentration level 15 min post-exercise and returning to baseline after $90 \mathrm{~min}$ [22].

That mTOR expression increased after a 7-day, not 3-day, period of whey protein intake indicates that the increased mTOR levels found in this study are 
different from the post-exercise transient rise in its levels induced by a bout of protein intake. The change in gene expression seen after 7 days of intake may be due to a constitutive alteration in muscles that is adaptably induced by the nutritional environment when a specific protein is continuously supplied.

A typical example of the nutrition-related constitutive alteration of whole-body metabolism is seen in incidences that became the basis for the Barker hypothesis [23] [24] [25], which is now recognized as the theory "developmental origin of health and disease (DOHaD)" [26]. The DOHaD theory states that the nutritional environments of fetuses and neonatal infants affect their adulthood metabolism and determine the levels of risk for developing lifestyle diseases. If fetuses and infants are exposed to environments deficient in energy and nutrients, they adapt their metabolic properties by modifying the expression patterns of metabolism-related genes, which are epigenetically retained through their lifespan. Because of this epigenetic alteration in metabolic patterns, they face difficulty adapting their metabolism to regular nutrition intake and have higher risks of lifestyle diseases, including cardiovascular and obesity-related diseases in adulthood.

A 7-day period of whey protein intake alters the expression patterns of mTOR and its related components. This finding implicates that the skeletal muscle may have an ability to adapt to nutritional environments, where protein species become a determinant factor. The adaptation of the muscle to whey protein observed in this study may involve the continuous enhancement of mechanisms recognizing protein species and regulating protein synthesis. In contrast, postexercise enhancement of protein synthesis by a bout of whey protein intake may be due to the transient activation of such mechanisms.

Exercise and dietary protein supplementation are the current standards in sarcopenia therapy. Although the mechanism of the metabolic adaptation to whey protein is not clear at this moment, the results in this study offer insight regarding supplementation with whey protein for dietary therapy in sarcopenia with a scientific basis for the superiority of whey protein. Consecutive-day intake of whey protein should be preferred to help protein metabolism in the muscles of patients with sarcopenia efficiently convert to anabolic metabolism, which may yield beneficial outcomes if it is combined with exercise.

\section{Conclusion}

This study describes that the consecutive-day intake of whey protein induces continuous upregulation of the mRNA and protein expression of mTOR in the resting gastrocnemius muscle of mice, indicating that it induces the continuous enhancement of protein synthesis in the resting skeletal muscle. This effect becomes evident when mice are fed whey protein for 7 consecutive days, but not 3 days. Long term intake of whey protein may cause constitutive adaptation of the skeletal muscle leading to the alteration in metabolic activities including protein synthesis. 


\section{Funding}

This work was supported by JSPS KAKENHI Grant Number JP15K00890.

\section{Conflicts of Interest}

The author declares no conflicts of interest regarding the publication of this paper.

\section{References}

[1] Rosenberg, I.H. (1989) Summary Comments. American Journal of Clinical Nutrition, 50, 1231-1233. https://doi.org/10.1093/ajcn/50.5.1231

[2] Walston, J.D. (2012) Sarcopenia in Older Adults. Current Opinion in Rheumatolo$g y, 24,623-627$. https://doi.org/10.1097/BOR.0b013e328358d59b

[3] Hoffmann, C. and Weigert, C. (2017) Skeletal Muscle as an Endocrine Organ: The Role of Myokines in Exercise Adaptations. Cold Spring Harbor Perspectives in Medicine, 7, pii: a029793. https://doi.org/10.1101/cshperspect.a029793

[4] Fried, L.P., Tangen, C.M., Walston, J., Newman, A.B., Hirsch, C., Gottdiener, J., Seeman, T., Tracy, R., Kop, W.J., Burke, G. and McBurnie, M.A. (2001) Cardiovascular Health Study Collaborative Research Group. Frailty in Older Adults: Evidence for a Phenotype. Journals of Gerontology. Series A, Biological Sciences and Medical Sciences, 56, M146-M156. https://doi.org/10.1093/gerona/56.3.M146

[5] Xue, Q.L., Bandeen-Roche, K., Varadhan, R., Zhou, J. and Fried, L.P. (2008) Initial Manifestations of Frailty Criteria and the Development of Frailty Phenotype in the Women's Health and Aging Study II. Journals of Gerontology. Series A, Biological Sciences and Medical Sciences, 63, 984-990. https://doi.org/10.1093/gerona/63.9.984

[6] Borst, S.E. (2004) Interventions for Sarcopenia and Muscle Weakness in Older People. Age and Ageing, 33, 548-555. https://doi.org/10.1093/ageing/afh201

[7] Perry, C.G.R., Lally, J., Holloway, G.P., Heigenhauser, G.J., Bonen, A. and Spriet, L.L. (2010) Repeated Transient mRNA Bursts Precede Increases in Transcriptional and Mitochondrial Proteins during Training in Human Skeletal Muscle. Journal of Physiology, 588, 4795-4810. https://doi.org/10.1113/jphysiol.2010.199448

[8] Saxton, R.A. and Sabatini, D.M. (2017) mTOR Signaling in Growth, Metabolism, and Disease. Cell, 168, 960-976. https://doi.org/10.1016/j.cell.2017.02.004

[9] Egan, B. and Zierath, J.R. (2013) Exercise Metabolism and the Molecular Regulation of Skeletal Muscle Adaptation. Cell Metabolism, 17, 162-184. https://doi.org/10.1016/j.cmet.2012.12.012

[10] Tang, J.E., Moore, D.R., Kujbida, G.W., Tarnopolsky, M.A. and Phillips, S.M. (2009) Ingestion of Whey Hydrolysate, Casein, or Soy Protein Isolate: Effects on Mixed Muscle Protein Synthesis at Rest and Following Resistance Exercise in Young Men. Journal of Applied Physiology, 107, 987-992. https://doi.org/10.1152/japplphysiol.00076.2009

[11] Burd, N.A., Yang, Y., Moore, D.R., Tang, J.E., Tarnopolsky, M.A. and Phillips, S.M. (2012) Greater Stimulation of Myofibrillar Protein Synthesis with Ingestion of Whey Protein Isolate v. Micellar Casein at Rest and after Resistance Exercise in Elderly Men. British Journal of Nutrition, 108, 958-962. https://doi.org/10.1017/S0007114511006271

[12] Kim, D.H., Sarbassov, D.D., Ali, S.M., King, J.E., Latek, R.R., Erdjument-Bromage, H., Tempst, P. and Sabatini, D.M. (2002) mTOR Interacts with Raptor to Form a 
Nutrient-Sensitive Complex That Signals to the Cell Growth Machinery. Cell, 110, 163-175. https://doi.org/10.1016/S0092-8674(02)00808-5

[13] Kim, D.H., Sarbassov, D.D., Ali, S.M., Latek, R.R., Guntur, K.V., Erdjument-Bromage, H., Tempst, P. and Sabatini, D.M. (2003) G $\beta$ L, a Positive Regulator of the Rapamycin-Sensitive Pathway Required for the Nutrient-Sensitive Interaction between Raptor and mTOR. Molecular Cell, 11, 895-904. https://doi.org/10.1016/S1097-2765(03)00114-X

[14] Hara, K., Maruki, Y., Long, X., Yoshino, K., Oshiro, N., Hidayat, S., Tokunaga, C., Avruch, J. and Yonezawa, K. (2002) Raptor, a Binding Partner of Target of Rapamycin (TOR), Mediates TOR Action. Cell, 110, 177-189. https://doi.org/10.1016/S0092-8674(02)00833-4

[15] Acosta-Jaquez, H.A., Keller, J.A., Foster, K.G., Ekim, B., Soliman, G.A., Feener, E.P., Ballif, B.A. and Fingar, D.C. (2009) Site-Specific mTOR Phosphorylation Promotes mTORC1-Mediated Signaling and Cell Growth. Molecular and Cellular Biology, 29, 4308-4324. https://doi.org/10.1128/MCB.01665-08

[16] Biolo, G., Maggi, S.P., Williams, B.D., Tipton, K.D. and Wolfe, R.R. (1995) Increased Rates of Muscle Protein Turnover and Amino Acid Transport after Resistance Exercise in Humans. American Journal of Physiology, 268, E514-E520. https://doi.org/10.1152/ajpendo.1995.268.3.E514

[17] Phillips, S.M., Tipton, K.D., Aarsland, A., Wolfe, S.E. and Wolfe, R.R. (1997) Mixed Muscle Protein Synthesis and Breakdown after Resistance Exercise in Humans. American Journal of Physiology, 273, E99-E107. https://doi.org/10.1152/ajpendo.1997.273.1.E99

[18] Phillips, S.M., Tipton, K.D., Ferrando, A.A. and Wolfe, R.R. (1999) Resistance Training Reduces the Acute Exercise-Induced Increase in Muscle Protein Turnover. American Journal of Physiology, 276, E118-E124. https://doi.org/10.1152/ajpendo.1999.276.1.E118

[19] Tipton, K.D., Elliott, T.A., Cree, M.G., Wolf, S.E., Sanford, A.P. and Wolfe, R.R. (2004) Ingestion of Casein and Whey Proteins Result in Muscle Anabolism after Resistance Exercise. Medicine and Science in Sports Exercise, 36, 2073-2081. https://doi.org/10.1249/01.MSS.0000147582.99810.C5

[20] Haraguchi, F.K., de Brito Magalhães, C.L., Neves, L.X., dos Santos, R.C., Pedrosa, M.L. and Silva, M.E. (2014) Whey Protein Modifies Gene Expression Related to Protein Metabolism Affecting Muscle Weight in Resistance-Exercised Rats. Nutrition, 30, 876-881. https://doi.org/10.1016/j.nut.2013.12.007

[21] Kumar, V., Selby, A., Rankin, D., Patel, R., Atherton, P., Hildebrandt, W., Williams, J., Smith, K., Seynnes, O., Hiscock, N. and Rennie, M.J. (2008) Age-Related Differences in the Dose-Response Relationship of Muscle Protein Synthesis to Resistance Exercise in Young and Old Men. Journal of Physiology, 587, 211-217. https://doi.org/10.1113/jphysiol.2008.164483

[22] Kraemer, W.J., Marchitelli, L., Gordon, S.E., Harman, E., Dziados, J.E., Mello, R., Frykman, P., McCurry, D. and Fleck, S.J. (1990) Hormonal and Growth Factor Responses to Heavy Resistance Exercise Protocols. Journal of Applied Physiology, 69, 1442-1450. https://doi.org/10.1152/jappl.1990.69.4.1442

[23] Barker, D.J. and Osmond, C. (1986) Infant Mortality, Childhood Nutrition, and Ischaemic Heart Disease in England and Wales. The Lancet, 1, 1077-1081. https://doi.org/10.1016/S0140-6736(86)91340-1

[24] Barker, D.J., Winter, P.D., Osmond, C., Margetts, B. and Simmonds, S.J. (1989) Weight in Infancy and Death from Ischaemic Heart Disease. The Lancet, 2, 577-580. 
https://doi.org/10.1016/S0140-6736(89)90710-1

[25] Barker, D.J., Gluckman, P.D., Godfrey, K.M., Harding, J.E., Owens, J.A. and Robinson, J.S. (1993) Fetal Nutrition and Cardiovascular Disease in Adult Life. The Lancet, 341, 938-941. https://doi.org/10.1016/0140-6736(93)91224-A

[26] Gluckman, P.D. and Hanson, M.A. (2004) Living with the Past: Evolution, Development, and Patterns of Disease. Science, 305, 1733-1736.

https://doi.org/10.1126/science.1095292 